\title{
Canine angiostrongylosis: recent advances in diagnosis, prevention, and treatment
}

This article was published in the following Dove Press journal:

Veterinary Medicine: Research and Reports

I December 2014

Number of times this article has been viewed

\section{Angela Di Cesare \\ Donato Traversa}

Faculty of Veterinary Medicine, University of Teramo, Teramo, Italy
Correspondence: Donato Traversa Faculty of Veterinary Medicine, University of Teramo, Piazza Aldo Moro 45, 64I00 Teramo, Italy Tel +390861266870 Fax +390861266873

Email dtraversa@unite.it
Abstract: Angiostrongylus vasorum is a parasitic nematode affecting the heart and pulmonary arteries of wild (eg, foxes) and domestic canids. The parasite has an indirect life cycle in which slugs and snails act as intermediate hosts. In the last few years the parasite has spread outside the traditional endemic foci, and there is a rise of documented cases of canine angiostrongylosis across Europe. Angiostrongylus vasorum causes cardiopulmonary disorders and coagulopathies, along with different nonspecific clinical signs. Fatal infections are frequently reported. Given the severity of the infection and the recent geographic spreading of the parasite, this article reviews and discusses the current knowledge of $A$. vasorum, with a special focus on recent insights on diagnosis, prevention, and treatment of dog angiostrongylosis.

Keywords: Angiostrongylus vasorum, dog, epidemiology, diagnostic approaches, control, therapy

\section{Introduction}

In the last decade the parasitic nematode Angiostrongylus vasorum (Nematoda, Metastrongyloidea) has greatly stimulated the attention of the scientific community, vet practitioners, and pharmaceutical companies due to its emergence in several areas and the major pathogenic role it plays in canine clinical practice.

Until a few years ago, our knowledge of dog angiostrongylosis was impaired by several gaps in epidemiology, diagnosis, treatment, and control of this disease. However, recent studies have answered different questions, especially on the geographic occurrence of $A$. vasorum, on reliable diagnostic approaches, and on effective therapeutic and preventive protocols.

The present review provides an overview of new insights and acquisitions achieved in the past decade on dog angiostrongylosis, with a special focus on diagnosis, treatment, and control. In addition, current epidemiological changes, possible changes in the impact of this parasite in canine medicine, and future perspectives in enhancing knowledge on $A$. vasorum are also illustrated and discussed.

\section{Biology and epidemiology}

The adult stages of $A$. vasorum reside in the cardiorespiratory system of infected hosts; ie, the heart and pulmonary arteries of dogs, wild canids (eg, foxes, wolves), and other animals. ${ }^{1,2}$ The parasite has an indirect life cycle involving a variety of mollusk slugs and snails as intermediate hosts, which are the source of infection for the vertebrates., ${ }^{3,4}$ The first stage larvae (L1) of the nematode are shed in the feces by infected dogs and mature to the third, infective, larval stage (L3) in the intermediate 
host. A dog becomes infected when it ingests the infected mollusk. ${ }^{3,4}$ Indeed, the actual mechanism of infection is not fully understood. It could be by direct predation of mollusks, but also by inadvertent ingestion (eg, scavenging on carrion or grass-chewing). A study has shown that L3 are capable to leave the gastropods, rendering themselves available to be swallowed directly, but the epidemiological importance of this route is unknown. ${ }^{5}$ In fact, in one study, ${ }^{5}$ L3 were stimulated in the water to experimentally leave a gastropod species (ie, the tropical aquatic snail Biomphalaria glabrata), which is an unnatural host of $A$. vasorum. ${ }^{2}$ Furthermore, the common frog (Rana temporaria) has been incriminated in the life cycle of $A$. vasorum as a paratenic and even intermediate host, ${ }^{6}$ but its actual biological role has not been unveiled thus far.

After ingestion, L3 penetrate the gut wall and migrate to the mesenteric lymph nodes, where they molt and develop up to the fifth larval stage, which then reach the right ventricle and pulmonary arteries through the mesenteric lymphatics, hepatic and portal veins, and caudal vena cava. Adult parasites mate in the pulmonary arteries and heart (mainly the right ventricle) of the host and the females produce eggs, which hatch quickly. L1 are carried to the lung capillaries, penetrate the alveolar and bronchial walls, reach the mucociliary escalator, are coughed up, ingested, and pass out in the feces. ${ }^{2,4}$

Ectopic locations of adult A. vasorum may include the pericardium, bladder, kidney, femoral artery, and anterior chamber of the eye, ${ }^{7-9}$ while L1 have also been recorded in the skin, diaphragm, liver, pancreas, central nervous system, and cerebrospinal fluid. ${ }^{10,11}$

Angiostrongylus vasorum has been traditionally considered to be prevalent mainly in well-isolated endemic geographic foci with a patchy distribution (ie, Southwestern France, Southern UK, and Denmark); only occasional or anecdotal cases have been reported in other regions until few years ago. ${ }^{4,6,12-14}$

There is the recent evidence of both an increase in the number of cases reported within known endemic foci (eg, Denmark and the $\mathrm{UK}^{2}$ ) and a spread beyond them, with the appearance of new foci in several regions previously free of infection. ${ }^{1,14-21}$ More specifically, since 2007-2008, A. vasorum has been described in dogs from Sweden, ${ }^{22}$ Greece, ${ }^{23}$ Italy, ${ }^{24}$ Switzerland, and Germany. ${ }^{12}$ In the UK, the nematode has spread from the area of original detection in the southern regions throughout the country, ${ }^{17}$ and progressively higher incidence rates have been recorded also in Germany, Denmark, and Italy. ${ }^{25-27}$ Recently, serological investigations have confirmed exposure to the parasite and the occurrence of active infections in dog populations from Germany, Poland, the UK, and Italy. ${ }^{19-21}$

In North America, A. vasorum has been found in dogs imported from Europe. ${ }^{28,29}$ A known endemic focus is located in the Atlantic Canadian provinces of Newfoundland and Labrador, which recently faced epidemiological changes similar to those concurrently occurring in Europe. ${ }^{30}$ In Newfoundland, the infection was confined only to the wild red fox population until recently, when $A$. vasorum has begun to be increasingly detected in domestic dogs as well. ${ }^{30}$

In 2011, the parasite was recorded in a red fox from West Virginia, USA, thus indicating a new geographical location for angiostrongylosis. In particular, this is the first autochthonous record of $A$. vasorum infection in the US and from outside of Newfoundland, Canada. ${ }^{31}$ Red foxes have the largest geographical distribution of any other carnivore in North America, ${ }^{32}$ where suitable susceptible definitive and intermediate hosts are abundant throughout the majority of this territory. ${ }^{30}$ Also, local climatic conditions guarantee a potential survival and spread of $A$. vasorum; thus, the likelihood that a spread of $A$. vasorum in mainland North America will become a reality in the near future is indeed high. ${ }^{30}$

The new reports of dog angiostrongylosis have raised the question of whether the nematode was indeed emerging in new areas or if an increased awareness has led to higher rates of diagnosis. The reasons for a possible emergence of $A$. vasorum have been considered speculative, but some drivers might be indeed involved in the spreading of $A$. vasorum from traditional foci to previously free areas. Climate changes have the potential to foster the distribution of a variety of intermediate and paratenic hosts and consequent modification of vector phenology, including gastropods..$^{13,33-36}$

As a key example, the increasing number of recent reports of mollusk-transmitted parasitoses has been suggested to result from the geographic spread of slugs and snails. ${ }^{14,37-41}$ Indeed, population dynamics and activity of different intermediate hosts, including mollusks, are sensitive to temperature and moisture, and the development of nematode larvae in invertebrates is also known to be temperature dependent. ${ }^{42}$

Despite the obvious species-specific differences among mollusk species, warmer winter temperatures and increased precipitation have the potential to favor the activity and reproduction of mollusks, thus nurturing the expansion of some species. ${ }^{43}$ Indeed, regional abundance, seasonal breeding, and periodic activity of slugs and snails may be influenced by climate changes. In particular, improvements of conditions for parasite development and for exposure 
of dogs to infected intermediate hosts is realistic. ${ }^{2}$ Helix aspersa, a land snail in which $A$. vasorum may develop to the infective stage, is present and abundant in those regions with a climate suitable for its development even in anthropized areas. ${ }^{44,45}$ After introductions into previously free areas for farming purposes or accidentally by the movement of plants and vegetables, ${ }^{46} H$. aspersa showed a rapid adaptation to a novel environment. ${ }^{46,47}$

The current climate changes may indeed play a role in the spreading of $A$. vasorum. In general, the parasite is vulnerable to low temperatures, and it is present only in those areas where the mean winter temperature never goes below $-4^{\circ} \mathrm{C} .{ }^{48}$ In the environment, $\mathrm{L} 1$ of $A$. vasorum die within 3 weeks after being exposed to $18^{\circ} \mathrm{C}-25^{\circ} \mathrm{C}$. ${ }^{49}$ Thus, mild and wet climates, and areas with humidity conditions suitable for the mollusks are the areas offering the best scenario for the life cycle of the parasite.

A climate envelope developed to investigate the geographic regions at risk of spread and/or invasion by $A$. vasorum has shown that several European regions (eg, central-northern areas of Europe and Italy) offer ecological and epidemiological conditions for the expansion of endemic foci of $A$. vasorum and the establishment of further new endemic foci. ${ }^{50}$

Pets traveling with their owners, international trade of goods and animals, and changes in the habitat of wild reservoirs and invertebrates may also be involved in the current epidemiological changes of dog angiostrongylosis.

The relevance of untreated subclinically infected dogs is very high, ${ }^{51,52}$ as an asymptomatic animal may introduce A. vasorum when it travels to nonendemic areas, as demonstrated in Australia, Newfoundland, and the US, even when dogs undergo rigorous screening (including parasitological analysis) and quarantine processes. ${ }^{28,53}$ Similarly, dogs traveling to endemic areas may introduce the parasite in free areas when they go home..$^{51,54}$

Reduction of woodlands has induced wild animals, especially foxes, to move into new hospitable environments (eg, the suburbs and cities) in order to look for anthropogenic food sources. These movements increase the contact between wildlife and pets, thus contributing to the spread of parasites harbored by wildlife. ${ }^{2,14,55}$ For instance, the high level of A. vasorum infection in foxes living in Denmark and UK and the high fox population density in the same area have been incriminated in the transmission of the parasite to the domestic dog. ${ }^{2,456}$ Interestingly, studies have demonstrated the existence of $A$. vasorum populations shared between different definitive hosts (ie, foxes, dogs, and wolves) in endemic regions. ${ }^{57-59}$
It is also true that lack of awareness and misdiagnosis could have resulted in underestimation and missed diagnosis in dogs. However, the high level of pathogenicity of angiostrongylosis makes it unlikely that many clinical cases in previous years were missed. Notwithstanding, the recent enhancement in terms of awareness of veterinary practitioners and improved diagnostic tools may have caused a rise in the number of diagnoses of a previously neglected parasite. This possibility is indeed realistic because specific parasitological techniques to detect $A$. vasorum larvae in the feces of infected animals are infrequently utilized in practice, ${ }^{60}$ and user-friendly rapid diagnostic kits have been marketed only very recently. ${ }^{61}$ However, different evidences indicate that this is an actually emerging parasite. For instance, in Italy $A$. vasorum was first reported in late 1980 s only in red foxes. ${ }^{62,63}$ The infection was likely confined to this wild host until recent years; the parasite has been recorded with increasing frequency in dogs since 2002 (Di Cesare et al, unpublished data, 2014). ${ }^{24,26,27,64-67}$ Despite increasing awareness among clinicians and researchers, which may lead a rise in documented diagnoses, the trend in higher incidence rates observed in other European countries and the concomitant expansion in foxes in several areas corroborate the hypothesis that the parasite is truly emerging and spreading. ${ }^{1,2,50}$

\section{Clinical features and diagnosis}

Studies have shown that dogs aged $<2$ years are more likely to be infected with $A$. vasorum than adult dogs, but the disease can occur at any age. ${ }^{27,68-71}$ The higher occurrence of angiostrongylosis in young subjects is likely due to age-related differences in dietary, playing, and scavenging behavior, and incomplete immune competency. There seems to be no gender or breed predisposition, although Cavalier King Charles' spaniels, Staffordshire bull terriers, Labrador retrievers, and hunting dogs have been frequently reported to be infected. ${ }^{51,70,72}$ The appearance of clinical signs is reported to be occasionally seasonal and the diagnosis of the disease is more common in winter and spring, ${ }^{25,69,72}$ due to the increased risk of infection in late summer (ie, the period when mollusks are most abundant and larval infections most mature). However, this pattern is weak, and clinical angiostrongylosis is observed at any time of year. ${ }^{2}$

The clinical scenario is due to the inflammation triggered by the presence of the parasite eggs and larval stages in the lungs and the damage caused by adult worms in the vessels. Especially in chronic infections, infiltration of inflammatory cells, disruption of alveolar architecture, thrombus formation, and fibrosis occur around larval parasites, thus 
suggesting that the host response to L1 penetration of the alveoli underlies lung pathogenesis. ${ }^{73}$ Nonetheless, a recent experimental study has demonstrated that respiratory distress may occur also in prepatent infections, given that lesions at the lungs were described before the appearance of L1 in the feces of the animals. ${ }^{74}$

In general, infected dogs present an obliterative thrombotic endarteritis and fibrosis with varying clinical signs. They can be absent or highly variable, ranging from minor to severe manifestations leading to death. ${ }^{27,51,60,70,71,75}$ If the infection is not treated, a progressive and potentially lifethreatening disease with a fatal outcome may occur.

Cardiopulmonary disorders and coagulopathies are the most important manifestations, but nonspecific clinical signs are frequently observed. Common respiratory signs include cough, dyspnea, and tachypnea due to bronchopneumonia. ${ }^{27,69-71}$ It is, however, worth mentioning that the absence of respiratory distress should not preclude inclusion of angiostrongylosis as a differential diagnosis. The severity of the disease depends on the number of parasites, with heavy burdens resulting in more acute pulmonary lesions and marked clinical signs..$^{6,70,76,77}$ The presence of adult worms in the arteries, with consequent pulmonary vessel thrombosis and vascular smooth muscle hypertrophy, may cause pulmonary hypertension and, eventually, right-sided cardiac dysfunction (cor pulmonale). Infected dogs may show congestive heart failure, including exercise intolerance, ascites, tachycardia, pale mucous membranes, severe dyspnea, syncope, and death. ${ }^{27,60,78-80}$

Other clinical manifestations, regardless of cardiovascular and/or respiratory involvement, are bleeding diathesis and neurological and gastrointestinal signs. ${ }^{2}$

Bleeding disorders and coagulopathies cause epistaxis, hemoptysis, postsurgical hematomas, gastrointestinal bleeding, hematuria, anemia, and petechial or ecchymotic hemorrhages in the conjunctiva, episclera, gingiva, and subcutis. ${ }^{70,81,82}$ Massive bleeding may occur in body cavities ${ }^{64}$ or into the brain or spinal cord. ${ }^{80}$

The origin of this bleeding diathesis is still to be clarified. A likely cause is a chronic low grade disseminated intravascular coagulation, with associated consumption of platelets and coagulation factors. In fact, infected dogs may show prolonged prothrombin and activated partial thromboplastin times and decreased factors V, VII, and VIII activities or alterations. ${ }^{71,83-85}$ Immune-mediated thrombocytopenia and von Willebrand's factor deficiency have been described ${ }^{86,87}$ and, in particular, thrombocytopenia has been described in naturally infected dogs that did not show alterations in specific prothrombin time and activated partial thromboplastin time, thus supporting the hypothesis of an immune-mediated thrombocyte destruction. ${ }^{71}$ Furthermore, this latter study supports the theory that thrombocytopathy and/or thrombocytopenia may indeed play a relevant role in most infected dogs showing hemorrhages. ${ }^{71}$

Dogs may present neurological manifestations due to embolism of larvae or eggs in the central nervous system ${ }^{29,73,88}$ or to intracranial, subdural bleeding. ${ }^{77,87,89}$ Depression, ataxia, seizures, ${ }^{12,82}$ central vestibular signs, forelimb or hind limb paralysis, and acute lumbar pain can be observed. ${ }^{29,75,77}$ Given that acute nontraumatic brain hemorrhages are uncommon in dogs, angiostrongylosis should be suspected as a cause in endemic areas. $^{79}$

Ocular lesions have also been recorded, with uveitis as the most common manifestation. ${ }^{7,29,90}$ Nonspecific gastrointestinal signs like vomiting, diarrhea, anorexia, and weight loss can also be present. ${ }^{27,70,75,82}$

In the aforementioned circumstances, it is clear that canine angiostrongylosis poses major diagnostic difficulties in vivo, as the clinical presentation is difficult to differentiate from other canine diseases; also, subclinical or atypical infections may occur.

A diagnosis of angiostrongylosis should be included in the differential in all dogs with appropriate and compatible clinical signs, regardless of their age. Nonetheless, the long prepatent period makes unlikely the occurrence of the disease in dogs aged less than 3-4 months. Additionally, the occurrence of subclinical infections renders frequent diagnostic screenings highly desirable in any endemic area.

At the clinical examination, thoracic auscultation may reveal increased or harsh lung sounds and crackles, ${ }^{70}$ and a systolic heart murmur due to tricuspid regurgitation can be heard. ${ }^{76,78}$ However, these findings are not pathognomonic of A. vasorum infection.

Different radiographic features have been described, usually correlated with the duration of the infection and the lesions caused by the parasite. At a glance, interstitial, bronchial, and alveolar radiographic patterns are all commonly described while vascular patterns are not, in both natural and experimental infections. ${ }^{91,92}$ Diffuse bronchial and interstitial pattern with focal alveolar infiltrates may be found in the early stage of the disease. In patent infections (ie, about 7 to 9 weeks' postinfection [PI]), a patchy alveolar pattern predominates, mainly at the periphery of the lung lobes, due to alveolar hemorrhage caused by migrating L1. ${ }^{91}$ Subsequently, until 21 weeks PI, alveolar changes become less prominent, with predominance of an interstitial pattern due to pulmonary 
consolidation and fibrosis. ${ }^{60}$ At the same time, right-side cardiac enlargement, dilatation of the pulmonary trunk, and truncated pulmonary arteries may be observed. ${ }^{91,92}$

Despite that these radiographic signs are not pathognomonic, a multifocal distribution and/or peripheral alveolar pattern $^{70,91,92}$ may suggest the occurrence of the parasitosis in animals with a compatible clinical scenario (Figure 1). Indeed, an alveolar infiltrate has been described in at least $50 \%$ of dogs examined in clinical case series, and its features (eg, diffuse, multifocal, or peripheral) depend upon the duration of infection. ${ }^{71,92}$ Also, interstitial infiltration is commonly observed in case series, and pleural fissures may be present in about the half of examined cases with several cases of concurrent interstitial pattern, which could reflect a chronicity of the infection. ${ }^{71}$ Interestingly, animals may show radiographic evidence of lung lesions despite presenting with no overt respiratory signs. Hence, the importance of thoracic radiography in investigating angiostrongylosis should not be disregarded even in the absence of cardiorespiratory signs. ${ }^{71}$

Computed tomography (CT) proved to be a useful tool for careful evaluation and definition of the pulmonary lesions associated with $A$. vasorum infection, but not for diagnosis of the infection. ${ }^{13} \mathrm{CT}$ findings suggestive of angiostrongylosis are multiple nodules merging to areas of consolidation, air bronchograms of varying extent, bronchial thickening, mild bronchiectasia, and enlarged lymph nodes. ${ }^{93}$

Magnetic resonance imaging is also a sensitive method for evaluating the extent of hemorrhages in the central nervous system, as shown in studies which described the features of brain and spinal cord hemorrhages in dogs with neurological clinical signs associated with angiostrongylosis. ${ }^{82,89}$

Both cor pulmonale and pulmonary hypertension may be diagnosed with echocardiography and Doppler echocardiography. Dogs infected with $A$. vasorum may present

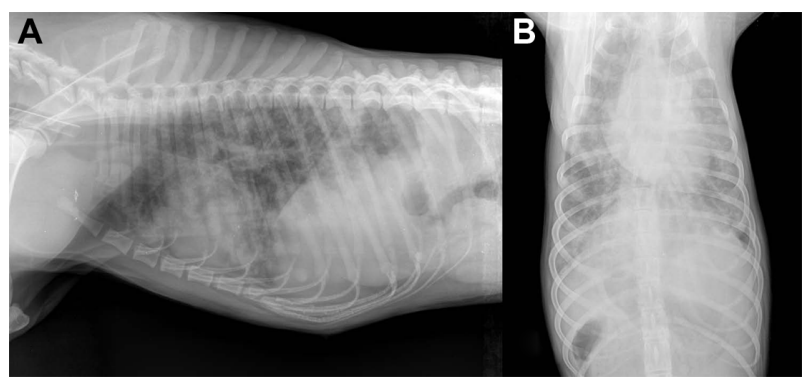

Figure I Laterolateral (A) and dorsoventral (B) view of thorax of an 18-month-old dog: moderate interstitial pattern mainly involving the caudodorsal lung fields and mild alveolar pattern with isolated fluffy infiltrates and a subtle right-sided cardiac enlargement (Courtesy of Dr Paolo Crisi, Teaching Veterinary Hospital, University of Teramo, Italy). right ventricular concentric and eccentric hypertrophy, systolic septal flattening, paradoxical septal motion, reduced left ventricular internal diameter, dilatation of pulmonary arteries, changes of the pulmonary flow profile, and high velocity tricuspid and pulmonary regurgitation..$^{24,60,77,78,94}$ Nevertheless, these findings are highly variable in infected dogs.

Not any hematological or biochemical changes are pathognomonic of angiostrongylosis. ${ }^{70,71}$ As an example, although eosinophilia may be present in infected dogs, its absence should not preclude consideration of angiostrongylosis in the differential diagnosis. ${ }^{71}$

L1 of $A$. vasorum may be detected in the feces using conventional copromicroscopy with direct fecal smears, floatation, and Baermann migration methods, with the latter being considered the technique of choice. ${ }^{95,96}$ Since free-living nematode larvae can be present in fecal samples, these should be collected rectally or just after emission to avoid any confusion in the identification of nematode larvae present in a sample. Moreover, A. vasorum L1 should be differentiated from those of other respiratory nematodes (Table 1) of dogs; ie, Crenosoma vulpis, Oslerus osleri, or Filaroides spp. ${ }^{72,77,97,98} \mathrm{~L} 1$ of $A$. vasorum (Figure 2) have a length of $310-400 \mu \mathrm{m}$, a width of $14-16 \mu \mathrm{m}$, an anterior cephalic button, a tip tail with a sinus wave curve, and a dorsal spine..$^{959-101}$ The length of $O$. osleri and Filaroides spp. L1 is inferior $(\sim 250 \mu \mathrm{m})$ to that of $A$. vasorum, they lack the prominent dorsal spine, and they have an S-shaped tail with a slight kink, while L1 of $C$. vulpis have a very pointed and straight tail and measure about 240-310 $\mu \mathrm{m} .^{97,102}$ L1 of $A$. vasorum should be also discriminated by those of Strongyloides stercoralis. One should also bear in mind that $S$. stercoralis may develop a free-living generation if the fecal sample is allowed to incubate; thus, the presence of adult stages does not definitively indicate that a contamination has occurred. ${ }^{14}$

The unpredictable presence of larvae and an inadequate sample size make direct fecal smear and floatation less sensitive than the Baermann method for detecting L1 of A. vasorum. Additionally, the reliability of floatation procedures to diagnose angiostrongylosis is influenced by the type of solution used, the time period of flotation, and the consequent dehydration of larvae, which may impair the results of the microscopic examination. ${ }^{14,95}$ It is worth mentioning that the high specific gravity of concentrated salt solutions renders larvae extremely difficult to detect and identify for osmotic damage. ${ }^{14,95}$

The positive hydro/thermotropism observed for live nematode larvae makes the Baermann migration method the 
Table I Differential features of nematode first stage larvae found in fecal samples of dogs.

\begin{tabular}{|c|c|c|}
\hline Nematode & Length ( $\mu \mathrm{m})$ & Caudal end \\
\hline $\begin{array}{l}\text { Angiostrongylus } \\
\text { vasorum }\end{array}$ & $310-400$ & $\begin{array}{l}\text { Tip with a dorsal spine and a } \\
\text { sinus wave curve }\end{array}$ \\
\hline Oslerus osleri & $\sim 250$ & Absence of dorsal spines and \\
\hline Filaroides spp. & & S-shaped end with a slight kink \\
\hline $\begin{array}{l}\text { Crenosoma } \\
\text { vulpis }\end{array}$ & $240-310$ & Pointed and straight tail \\
\hline $\begin{array}{l}\text { Strongyloides } \\
\text { stercoralis }\end{array}$ & $150-390$ & Pointed and straight tail \\
\hline
\end{tabular}

Note: Adapted from Traversa D, Di Cesare A, Conboy G. Canine and feline cardiopulmonary parasitic nematodes in Europe: emerging and underestimated. Parasit Vectors. 2010;3:62. ${ }^{14}$ http://creativecommons.org/licenses/by/2.0/.

gold standard to diagnose angiostrongylosis. Nonetheless, this test is relatively time-consuming ( $8-36$ hours), requires operator skills, and has inherent shortcomings, such as the inability to detect the parasite during the prepatent period and when larvae are not being shed, even in the presence of severe clinical signs. The prepatent period of $A$. vasorum is long and variable, ranging from 1 to 4 months, although the patency usually begins between $\sim 40-60$ days after infection. ${ }^{6,13,100,103}$

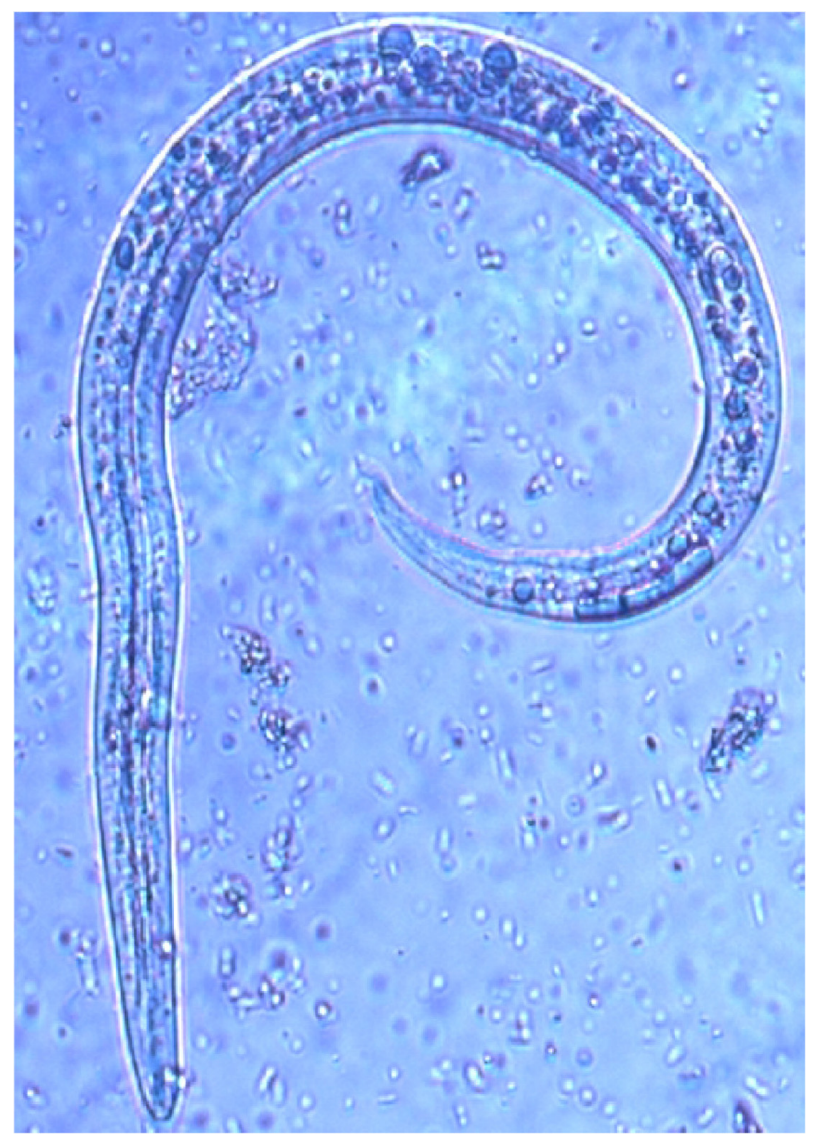

Figure 2 Baermann test: Angiostrongylus vasorum, first stage larva.

Note: Adapted from Traversa D, Di Cesare A, Conboy G. Canine and feline cardiopulmonary parasitic nematodes in Europe: emerging and underestimated. Parasit Vectors. 2010;3:62. ${ }^{14}$ http://creativecommons.org/licenses/by/2.0/.
This variable time-lapse may indeed influence the reliability of the Baermann method, which also is impaired by the intermittent shedding of $\mathrm{L} 1$ in patent infections. It has been experimentally shown that dogs may present irregular larval shedding pattern with periods of negative fecal results and of inconsistent shedding. Additionally, peaks of larval shedding are considerably variable in animals infected for the first time (at about 12 and 38 weeks PI) and in reinfected animals (at about 17 and 30 weeks PI) as well. ${ }^{88}$ Therefore, repeated examinations may be necessary to increase the sensitivity of the Baermann examination. ${ }^{14,77}$

Alternative diagnostic approaches consist in the detection of L1 in tracheal swabs or washes, bronchoalveolar lavage, or fine needle lung aspirates..$^{53,70,104}$ However, these techniques are used rarely, may be invasive, have potential risks, and may fail in detecting nematode larvae due to absence of significant pulmonary tissue involvement, prepatent infections, or poor sample recovery. ${ }^{70}$ Moreover, angiostrongylosis may be diagnosed with cytological smears from the hemothorax. ${ }^{64}$ In the case of larval dissemination, microscopic examination of urinary sediment in routine urinalysis may allow the diagnosis of the disease. ${ }^{13}$

Various serological techniques have been evaluated for diagnosing A. vasorum infection, but in-clinic devices have been developed and marketed only recently.

Sera examined with an enzyme-linked immunosorbent assay (ELISA), evaluated since the 1990s, showed a specific antibody response and increasing ELISA titer values, depending upon the humoral response in experimental infected animals. ${ }^{105}$ Later, the same authors provided evidence that the Western blot had a higher sensitivity than ELISA in the detection of specific antibodies against three principal antigens in A. vasorum proteins. However, the specificity of this test was not ultimately verified. ${ }^{106}$

A recent study investigated the usefulness of $\mathrm{L} 1$ and adult $A$. vasorum proteins in immunoblotting assays using sera of experimentally and naturally infected dogs. While the crude extract of $A$. vasorum adults shared antigens with other parasites, thus resulting in crossreactions, four proteins in dogs with $A$. vasorum were recognized by immunoglobin $\mathrm{G}$ antibodies that had no cross-reaction with sera of dogs naturally infected with other helminthes. ${ }^{107}$

A sandwich ELISA detecting circulating antigens in sera from infected dogs showed a sensitivity of $92 \%$ and scored negative results when applied on sera collected from dogs coming from nonendemic areas and from dogs infected only with the lungworm C. vulpis. ${ }^{108}$ 
Other highly specific ELISAs have recently proved to be powerful and affordable for the diagnosis of the infection in both individual and population studies. ${ }^{109,110}$ Specifically, the presence of circulating $A$. vasorum antigens may be detected with a sandwich ELISA using mono- and polyclonal antibodies with a sensitivity and specificity of $95.7 \%$ and $94 \%$, respectively. ${ }^{109}$ Specific antibodies may be detected with an ELISA based on excretory/secretory antigens of adult $A$. vasorum, with a sensitivity and specificity of $85.7 \%$ and $98.8 \%$, respectively. ${ }^{110}$ The interpretation of these two ELISAs differs because specific antibodies indicate the parasite exposure, while antigen detection represents an actual infection. ${ }^{109}$ Animals scoring positive upon both tests should be considered to be harboring an active $A$. vasorum infection, while dogs positive only for the presence of antibodies may have become infected recently, in that they are present at 3 weeks PI while the antigen is first detected 5 weeks PI. ${ }^{109,110}$ It should be taken into account that dogs seropositive for antibodies may also be free of parasites, for instance after a self-curing or after a purposed or serendipitous administration of a broad-spectrum macrolactone effective against A. vasorum. On the other hand, animals become negative for antigens within 16-34 days after an effective anthelmintic administration (eg, spot-on containing moxidectin), ${ }^{109}$ while antibodies persist up to 63 days after treatment. ${ }^{110}$

In the last year, a rapid in-clinic assay (Angio Detect ${ }^{\mathrm{TM}}$ Test, IDEXX Laboratories) (Figure 3) has been marketed for the serological detection of the circulating antigen of A. vasorum, aiming at a routine in-clinic diagnostic use. The sensitivity of this assay was investigated against sera from dogs with a natural infection, while the specificity was evaluated using samples from dogs infected by other single or mixed parasitoses. ${ }^{61}$ This rapid device showed a good sensitivity and a very high specificity; thus, it represents a cost-effective, user-friendly, and useful tool to be applied in veterinary practices. Baermann-positive animals may score negative on this assay, but in general it allows a rapid detection of infected dogs, with or without clinical signs..$^{52,61}$ This is of importance considering that an early diagnosis is key for initiating an effective treatment and that angiostrongylosis may be fatal if left untreated.

With regard to deoxyribonucleic acid (DNA)-based methods, some approaches have been recently developed to overcome the constraints of classical diagnostics. First, the characterization of the mitochondrial DNA (mtDNA) gene encoding for the cytochrome $\mathrm{c}$ oxidase subunit 1 of A. vasorum provided diagnostic genetic markers. Based on polymerase chain reaction (PCR) restriction fragment

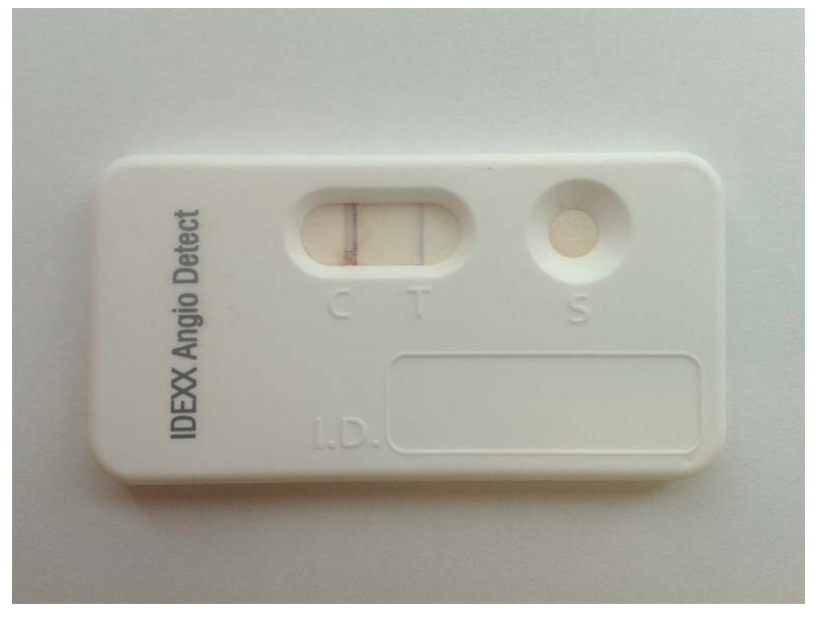

Figure 3 Positive result of the rapid in-clinic assay Angio Detect ${ }^{\mathrm{TM}}$ Test (IDEXX Laboratories).

length polymorphism analysis of this gene, $A$. vasorum can be differentiated from other closely related species (ie, Angiostrongylus cantonensis and Angiostrongylus costaricensis). ${ }^{111}$ Second, a real-time PCR specific for regions within the ribosomal DNA of $A$. vasorum has been validated to amplify parasite DNA from both definitive and intermediate host samples. This genetic test proved able to amplify a single $\mathrm{L} 1 \mathrm{in} 200 \mu \mathrm{L}$ of blood and in $200 \mathrm{mg}$ of feces, thus showing a lower limit of detection than the Baermann method. ${ }^{112}$ PCRs have been also be successful for detecting and identifying L1 in feces, ${ }^{16}$ and for diagnosing the disease from fecal ${ }^{112}$ and lung samples. ${ }^{80}$

\section{Treatment and control}

Different therapeutic options have been exploited for angiostrongylosis and benzimidazoles, and macrocyclic lactones have been successfully used in different formulations to cure the infection.

A spot-on formulation containing the neonicotinoid ectoparasiticide imidacloprid $10 \%$ and the endoectoparasiticide macrolactone moxidectin $2.5 \%$ was shown to be effective for both therapy and prevention of canine angiostrongylosis. For treating A. vasorum, moxidectin present in this formulation demonstrated, with a single application, an efficacy $(85.2 \%)$ similar to that observed in dogs treated with fenbendazole (91.3\%) daily at $25 \mathrm{mg} / \mathrm{kg}$ for 20 days, ${ }^{113}$ and overlapping the efficacy $(84.8 \%)$ achieved previously using milbemycin oxime $(0.5 \mathrm{mg} / \mathrm{kg})$ given orally once a week for 4 weeks. ${ }^{114}$ Also, a single additional treatment with moxidectin is able to stop the larval shedding in dogs which are still infected after the first treatment, along with radiological improvements. ${ }^{27,113}$ 
Infected dogs may require a supportive treatment based on their clinical scenario. Oxygen supplementation should be given in dogs with respiratory compromise, and antibiotics may be administered in cases of secondary bacterial infections, though this is not routinely indicated. ${ }^{2,78,94}$ Dyspneic dogs should be confined in cages, and should receive diuretics, bronchodilators, and oxygen-enriched air. ${ }^{13}$

Dogs may receive corticosteroids at immunosuppressive doses for treating immune-mediated thrombocytopenia or for preventing anaphylactic reactions post-treatment with a parasiticide. ${ }^{1,86,115}$ Indeed, the frequency of posttreatment anaphylaxis in treating angiostrongylosis is still unknown.

Transfusion of whole blood may be recommended in dogs suffering of hemorrhagic diathesis to replace erythrocytes and to provide coagulation factors. When not available, packed red blood cells and fresh frozen plasma are useful alternatives. ${ }^{1}$ Cardiac dysfunctions may be controlled with the administration of angiotensin-converting enzyme inhibitors and diuretics, or both. .,78 $^{2}$

With regard to chemoprevention, the imidacloprid $10 \%$ /moxidectin $2.5 \%$ spot-on treatment showed an effective and safe larvicidal activity in experimentally infected dogs. ${ }^{74}$ Indeed, a single topical application is $100 \%$ effective in the elimination of fourth-stage larvae and preadults of the parasite, thus preventing patent infections. This use has the potential to prevent or at least minimize the onset of severe cardiopulmonary tissue lesions in infected dogs. In practical terms, a monthly anthelmintic administration with this formulation is powerful for preventing the establishment of adult stages of $A$. vasorum and the clinical onset of the disease, and it is recommended especially for dogs living in endemic areas. $^{74}$

Milbemycin oxime showed an efficacy of $85 \%$ for prevention of canine angiostrongylosis when administered at $0.5 \mathrm{mg} / \mathrm{kg} 30$ and 60 days after experimental infection with A. vasorum. ${ }^{114}$

The efficacy of a combination containing the tetracyclic macrolide insecticide spinosad and milbemycin oxime was evaluated in the prevention of experimental canine angiostrongylosis. This combination tablet was administered at the lower end of the expected label dose range for the formulation, and the macrolactone contained proved to be highly efficacious $(>98 \%)$ in preventing the development of adult $A$. vasorum and in reducing the lung damage induced by the parasite. As such, these chewable tablets, given monthly, have the potential to prevent dogs from developing clinical or subclinical angiostrongylosis. ${ }^{116}$
Direct prophylactic measures in endemic areas should rely on daily disposal of feces of dogs to minimize environmental accumulation of larvae, and on education and awareness on risks of angiostrongylosis.

Pet owners should be aware that young animals are more prone to be infected and that high care levels should be maintained when animals play, roam, and scavenge in areas where slugs and snails are present. A direct control of mollusks is, of course, unfeasible for the severe toxicologic risks of common molluscicides, which certainly outweigh the benefits achievable in terms of reduced risk of infection. ${ }^{1}$ In particular, the use of environmental molluscicides does not guarantee a true reduction in gastropod populations; they are also dangerous for the environment and for the dogs. In fact, the use of these toxic chemicals could increase dogs' access to dead slugs and snails harboring viable L3. ${ }^{2}$

A decrease in L1 availability to intermediate hosts through biological control with predatory fungi has been evaluated ${ }^{117}$ but at the moment it is not known to which extent this approach may be used in the field.

In known endemic areas, prevention of angiostrongylosis should rely not only on direct prophylactic measures and on the administration of macrocyclic lactones when appropriate, but also on routine screening of dogs through routine copromicroscopic examinations or serological assays. In this way, these dogs may be treated to cure the infection and interrupt the biological cycle of the parasite.

\section{Innovative insights}

The characterization of the mitochondrial genome of A. vasorum provided a source of markers for studying the population genetics and epidemiology of the nematode. Molecular tools targeting specific mtDNA markers could be useful for further investigations into understanding host specificity and studying the epidemiology of the parasite in different countries. In fact, genetic variations in $A$. vasorum populations have already been described ${ }^{57,112}$ and, with the availability of the complete mtDNA, further studies may be carried out to evaluate if affiliations of particular genetic variants to different invertebrate and/or vertebrate hosts exist or not. These mitochondrial markers may be powerful to understand whether the different clinical forms of angiostrongylosis are caused by specific genetic variants of A. vasorum. ${ }^{118}$

The recent exploration of the $A$. vasorum transcriptome predicted molecules essential in metabolic and biological processes, in host-immune responses as well as in drug targets and drug candidates. The most highly transcribed 
molecules are contigs which encode for excretory/secretory and immunomodulatory proteins. Also, potential targets for anthelmintic treatment (ie, neurotransmitter receptors, a chemosensory ion channel, and cysteine proteinase-3, and proteins likely to be targeted in vaccine intervention) were also predicted. ${ }^{119}$ Further studies are warranted to investigate the practical implications of these achievements.

\section{Concluding remarks}

Angiostrongylus vasorum is an emerging pathogen of dogs, spreading from known endemic foci to other areas. Thus, this parasite is gaining more and more attention in veterinary medicine and, if the current trends in epidemiological changes continue, it is clear that in the near future we will face an increasing number of clinical cases in several geographic areas.

Practitioners should be vigilant for $A$. vasorum infection, especially in dogs from endemic areas but also in those pets which live in regions considered free of infections. In fact, the nematode may be easily introduced in free territories; thus, the disease should not be ruled out in the presence of compatible clinical signs or even in apparently healthy dogs living in regions where the parasite is considered absent. In fact, given the likely further geographical spread of $A$. vasorum, practitioners in areas at the moment not considered endemic should be highly conscious of the importance of dog angiostrongylosis.

From a practical standpoint, the use of compounds active against $A$. vasorum is indeed of importance. Moxidectin is powerful in both treating and preventing the infection with high efficacy and, interestingly, it has also been shown that in endemic areas dogs treated every 3 months with milbemycin oxime are half as likely to be infected as those given fenbendazole or not treated. ${ }^{69}$

The availability of macrocylic lactones for the chemoprevention of dog angiostrongylosis opens new avenues for the control of this disease. However, a risky season toward a seasonal chemoprevention is difficult to identify as, for instance, for Dirofilaria immitis. Thus, recommendations for chemoprevention must take each local epidemiological situation into appropriate account. ${ }^{2}$

Despite the fact that treatment and prevention are straightforward, the disease can be problematic to diagnose even in the presence of clinical signs. The different clinical scenarios associated with infection are well known, and certain clinical signs should always set off alarm bells for clinicians working in endemic areas or with traveling pets. Any suspected dog should undergo appropriate diagnosis; in endemic areas, healthy dogs should also be screened regularly for $A$. vasorum. The gold standard Baermann method of screening suffers from inherent drawbacks, despite the fact that it presents the highest sensitivity values in conventional clinical settings. The recently developed serological rapid test provides new opportunities for the diagnosis of the disease and for routine screenings in both endemic and free areas, even in the absence of clinical signs. In fact, this device can be directly used in veterinary practices and it represents a valid tool for a quick diagnosis. ${ }^{61}$ On the other hand, it should be always borne in mind that dogs presenting clinical signs compatible with angiostrongylosis but negative at the Angio Detect ${ }^{\mathrm{TM}}$ Test should be examined with the Baermann method before ruling out the infection, especially in endemic areas.

A continuous awareness of new methods available for diagnosis and of protocols for treating and preventing the disease is crucial to reduce the current spread of this lifethreatening nematode of dogs.

\section{Disclosure}

The authors report no conflicts of interest in this work.

\section{References}

1. Helm JR, Morgan ER, Jackson MW, Wotton P, Bell R. Canine angiostrongylosis: an emerging disease in Europe. $J$ Vet Emerg Crit Care (San Antonio). 2010;20(1):98-109.

2. Morgan E, Shaw S. Angiostrongylus vasorum infection in dogs: continuing spread and developments in diagnosis and treatment. J Small Anim Pract. 2010;51(12):616-621.

3. Anderson RC. The Superfamily Metastrongyloidea. In: Nematode Parasites of Vertebrates. Their Development and Transmission. 2nd ed, CABI Publishing, Guilford, UK; 2000:162-163.

4. Morgan ER, Shaw SE, Brennan SF, De Waal TD, Jones BR, Mulcahy G. Angiostrongylus vasorum: a real heartbreaker. Trends Parasitol. 2005;21(2):49-51.

5. Barçante TA, Barçante JM, Dias SR, Lima Wdos S. Angiostrongylus vasorum (Baillet, 1866) Kamensky, 1905: emergence of third-stage larvae from infected Biomphalaria glabrata snails. Parasitol Res. 2003;91(6):471-475.

6. Bolt G, Monrad J, Koch J, Jensen AL. Canine angiostrongylosis: a review. Vet Rec. 1994;135(19):447-452.

7. Rosenlund P, Boserup F, Monrad J. Angiostrongylus vasorum in the anterior chamber of the eye in dogs. Eur J Compan Anim Pract. 1993;3: 31-33.

8. King MCA, Grose RMR, Startup G. Angiostrongylus vasorum in the anterior chamber of a dog's eye. J Small Anim Pract. 1994;35(6): 326-328.

9. Cury MC, Lima WS. Rupture of femoral artery in a dog infected with Angiostrongylus vasorum. Vet Parasitol. 1996;65(3-4):313-315.

10. Oliveira-Júnior SD, Barçante JM, Barçante TA, Ribeiro VM, Lima WS. Ectopic location of adult worms and first-stage larvae of Angiostrongylus vasorum in an infected dog. Vet Parasitol. 2004;121(3-4):293-296.

11. Lepri E, Veronesi F, Traversa D, et al. Disseminated angiostrongylosis with massive cardiac and cerebral involvement in a dog from Italy. Parasitol Res. 2011;109(2):505-508.

12. Staebler S, Ochs H, Steffen F, et al. Autochthonous infections with Angiostrongylus vasorum in dogs in Switzerland and Germany. Schweiz Arch Tierheilkd. 2005;147(3):121-127. German.

13. Koch J, Willesen JL. Canine pulmonary angiostrongylosis: an update. Vet J. 2009;179(3):348-359. 
14. Traversa D, Di Cesare A, Conboy G. Canine and feline cardiopulmonary parasitic nematodes in Europe: emerging and underestimated. Parasit Vectors. 2010;3:62.

15. Morgan ER, Tomlinson A, Hunter S, et al. Angiostrongylus vasorum and Eucoleus aerophilus in foxes (Vulpes vulpes) in Great Britain. Vet Parasitol. 2008;154(1-2):48-57.

16. Helm J, Gilleard JS, Jackson M, Redman E, Bell R. A case of canine Angiostrongylus vasorum in Scotland confirmed by PCR and sequence analysis. J Small Anim Pract. 2009;50(5):255-259.

17. Yamakawa Y, McGarry JW, Denk D, et al. Emerging canine angiostrongylosis in northern England: five fatal cases. Vet Rec. 2009; 164(5):149-152.

18. Hurníková Z, Miterpáková M, Mandelík R. First autochthonous case of canine Angiostrongylus vasorum in Slovakia. Parasitol Res. 2013;112(10):3505-3508.

19. Guardone L, Schnyder M, Macchioni F, Deplazes P, Magi M. Serological detection of circulating Angiostrongylus vasorum antigen and specific antibodies in dogs from central and northern Italy. Vet Parasitol. 2013;192(1-3):192-198.

20. Schnyder M, Schaper R, Pantchev N, Kowalska D, Szwedko A, Deplazes P. Serological detection of circulating Angiostrongylus vasorum antigen- and parasite-specific antibodies in dogs from Poland. Parasitol Res. 2013;112 Suppl 1:109-117.

21. Schnyder M, Schaper R, Bilbrough G, Morgan ER, Deplazes P. Seroepidemiological survey for canine angiostrongylosis in dogs from Germany and the UK using combined detection of Angiostrongylus vasorum antigen and specific antibodies. Parasitology. 2013; 140(11):1442-1450.

22. Ablad B, Christensson D, Lind EO, Agren E, Morner T. Angiostrongylus vasorum etablerad i Sverige. Svensk Veterinartidning. 2003;12:11-15. Swedish.

23. Papazahariadou M, Founta A, Papadopoulos E, Chliounakis S, Antoniadou-Sotiriadou K, Theodorides Y. Gastrointestinal parasites of shepherd and hunting dogs in the Serres Prefecture, Northern Greece. Vet Parasitol. 2007;148(2):170-173.

24. Traversa D, Torbidone A, Malatesta D, Guglielmini C. Occurrence of fatal canine Angiostrongylus vasorum infection in Italy. Vet Parasitol. 2008;152(1-2):162-166.

25. Taubert A, Pantchev N, Vrhovec MG, Bauer C, Hermosilla C. Lungworm infections (Angiostrongylus vasorum, Crenosoma vulpis, Aelurostrongylus abstrusus) in dogs and cats in Germany and Denmark in 2003-2007. Vet Parasitol. 2009;159(2):175-180.

26. Di Cesare A, Castagna G, Meloni S, et al. Canine and feline infections by cardiopulmonary nematodes in central and southern Italy. Parasitol Res. 2011;109 Suppl 1:S87-S96.

27. Traversa D, Di Cesare A, Meloni S, et al. Canine angiostrongylosis in Italy: occurrence of Angiostrongylus vasorum in dogs with compatible clinical pictures. Parasitol Res. 2013;112(7):2473-2480.

28. Williams JF, Lindemann B, Padgett GA, Smith OL. Angiostrongylosis in a greyhound. J Am Vet Med Assoc. 1985;186(10):1101-1103.

29. Perry AW, Hertling R, Kennedy MJ. Angiostrongylosis with disseminated larval infection associated with signs of ocular and nervous disease in an imported dog. Can Vet J. 1991;32(7):430-431.

30. Conboy GA. Canine angiostrongylosis: the French heartworm: an emerging threat in North America. Vet Parasitol. 2011;176(4):382-389.

31. Kistler WM, Brown JD, Allison AB, Nemeth NM, Yabsley MJ. First report of Angiostrongylus vasorum and Hepatozoon from a red fox (Vulpes vulpes) from West Virginia, USA. Vet Parasitol. 2014; 200(1-2):216-220.

32. Kamler JF, Ballard WB. A review on native and non-native red foxes in North America. Wildlife Soc Bull. 2002;30:370-379.

33. Jobin W. Dams and Disease: Ecological Design and Health Impacts of Large Dams, Canals, and Irrigation Systems. London, New York: E\&FN Spon; 1999.

34. Xu XJ, Yang XX, Dai YH, Yu GY, Chen LY, Su ZM. Impact of environmental change and schistosomiasis transmission in the middle reaches of the Yangtze River following the Three Gorges construction project. Southeast Asian J Trop Med Public Health. 1999;30(3):549-555.
35. Root TL, Price JT, Hall KR, Schneider SH, Rosenzweig C, Pounds JA. Fingerprints of global warming on wild animals and plants. Nature. 2003;421(6918):57-60.

36. Pedersen UB, Midzi N, Mduluza T, et al. Modelling spatial distribution of snails transmitting parasitic worms with importance to human and animal health and analysis of distributional changes in relation to climate. Geospat Health. 2014;8(2):335-343.

37. Macpherson CN. Human behaviour and the epidemiology of parasitic zoonoses. Int J Parasitol. 2005;35(11-12):1319-1331.

38. Patz JA, Graczyk TK, Geller N, Vittor AY. Effects of environmental change on emerging parasitic diseases. Int J Parasitol. 2000;30(12-13): 1395-1405

39. Prociv P, Carlisle MS. The spread of Angiostrongylus cantonensis in Australia. Southeast Asian J Trop Med Public Health. 2001; 32 Suppl 2:126-128.

40. Tomlinson AJ, Taylor M, Roberts E. Angiostrongylus vasorum in canids. Vet Rec. 2006;159:160.

41. Di Cesare A, Crisi PE, Di Giulio E, et al. Larval development of the feline lungworm Aelurostrongylus abstrusus in Helix aspersa. Parasitol Res. 2013;112(9):3101-3108.

42. Petrić D, Zgomba M, Bellini R, Becker N. Surveillance of mosquito populations: a key element to understanding the spread of invasive vector species and vector-borne diseases in Europe. In: Mihailović D, editor. Essays on Fundamental and Applied Environmental Topics. Hauppauge, NY: Nova Science Publishers; 2012:193-224.

43. Willis JC, Bohan DA, Choi YH, Conrad KF, Semenova MA. Use of an individual-based model to forecast the effect of climate change on the dynamics, abundance and geographical range of the pest slug Deroceras reticulatum in the UK. Global Change Biol. 2006;12(9): 1643-1657.

44. Guiller A, Madec L. Historical biogeography of the land snail Cornu aspersum: a new scenario inferred from haplotype distribution in the Western Mediterranean basin. BMC Evol Biol. 2010;10:18.

45. Ansart A, Guiller A, Madec L. Invasive Species Compendium: Cornu Aspersum. CABI editor: London, UK; 2009.

46. Guiller A, Martin MC, Hiraux C, Madec L. Tracing the invasion of the mediterranean land snail Cornu aspersum aspersum becoming an agricultural and garden pest in areas recently introduced. PLoS One. 2012;7(12):e49674.

47. Madec L, Desbuquois C, Coutellec-Vreto MA. Phenotypic plasticity in reproductive traits: importance in the life history of Helix aspersa (Mollusca: Helicidae) in a recently colonized habitat. Biol J Linn Soc. 2000;69(1):25-39.

48. Jeffery RA, Lankester MW, McGrath MJ, Whitney HG. Angiostrongylus vasorum and Crenosoma vulpis in red foxes (Vulpes vulpes) in Newfoundland, Canada. Can J Zool. 2004;82(1):66-74.

49. Bordeau P. Canine Angiostrongylus vasorum infestation. Recueil de Medicine Veterinaire. 1993;169(5-6):401-407.

50. Morgan ER, Jefferies R, Krajewski M, Ward P, Shaw SE. Canine pulmonary angiostrongylosis: the influence of climate on parasite distribution. Parasitol Int. 2009;58(4):406-410.

51. Al-Sabi MN, Kapel CM, Johansson A, Espersen MC, Koch J, Willesen JL. A coprological investigation of gastrointestinal and cardiopulmonary parasites in hunting dogs in Denmark. Vet Parasitol. 2013;196(3-4):366-372.

52. Di Cesare A, Carlo M, Venco L, Pampurini F, Centaro E, Traversa D. Subclinical Angiostrongylus vasorum infection in a terrier dog kennel. Pol J Nat Sci. 2014; In press.

53. Tebb AI, Johnson VS, Irwin PJ. Angiostrongylus vasorum (French heartworm) in a dog imported into Australia. Aust Vet J. 2007;85(1-2):23-28.

54. Finnerup E. Angiostrongylus vasorum in dog: a survey and case history (Angiostrongylus vasorum hos hund: En oversigt og en kasuistik). Dansk Vet Tidsskr. 1983;66:338-340.

55. Deplazes P, Hegglin D, Gloor S, Romig T. Wilderness in the city: the urbanization of Echinococcus multilocularis. Trends Parasitol. 2004;20(2):77-84. 
56. Saeed I, Maddox-Hyttel C, Monrad J, Kapel CM. Helminths of red foxes (Vulpes vulpes) in Denmark. Vet Parasitol. 2006;139(1-3): $168-179$.

57. Jefferies R, Shaw SE, Willesen J, Viney ME, Morgan ER. Elucidating the spread of the emerging canid nematode Angiostrongylus vasorum between Palaearctic and Nearctic ecozones. Infect Genet Evol. 2010;10(4):561-568.

58. Eleni C, De Liberato C, Azam D, Morgan ER, Traversa D. Angiostrongylus vasorum in wolves in Italy. Int $J$ Parasitol Parasites Wildl. 2014;3(1):12-14.

59. Eleni C, Grifoni G, Di Egidio A, Meoli R, De Liberato C. Pathological findings of Angiostrongylus vasorum infection in red foxes (Vulpes vulpes) from Central Italy, with the first report of a disseminated infection in this host species. Parasitol Res. 2014;113(3):1247-1250.

60. Traversa D, Guglielmini C. Feline aelurostrongylosis and canine angiostrongylosis: a challenging diagnosis for two emerging verminous pneumonia infections. Vet Parasitol. 2008;157(3-4):163-174.

61. Schnyder M, Stebler K, Naucke TJ, Lorentz S, Deplazes P. Evaluation of a rapid device for serological in-clinic diagnosis of canine angiostrongylosis. Parasit Vectors. 2014;7:72.

62. Poli A, Arispici M, Marconcini A, Mancianti F, de Monte D. Angiostrongylus vasorum (Baillet, 1866) in red foxes (Vulpes vulpes L.) in Italy. J Wildl Dis. 1984;20(4):345-346.

63. Poli A, Arispici M, Mancianti F, Abramo F. Pathology of naturally acquired Angiostrongylus vasorum infection in the red fox (Vulpes vulpes). Angew Parasitol. 1991;32(3):121-126.

64. Sasanelli M, Paradies P, Otranto D, Lia RP, de Caprariis D. Haemothorax associated with Angiostrongylus vasorum infection in a dog. J Small Anim Pract. 2008;49(8):417-420.

65. Tieri E, Pomilio F, Di Francesco G, et al. Angiostrongylus vasorum in 20 dogs in the province of Chieti, Italy. Vet Ital. 2011;47(1):77-88, 65.

66. Riggio F, Mannella R, Ariti G, Perrucci S. Intestinal and lung parasites in owned dogs and cats from central Italy. Vet Parasitol. 2013;193(1-3): 78-84.

67. Pipia AP, Varcasia A, Tosciri G, et al. New insights onto cardiopulmonary nematodes of dogs in Sardinia, Italy. Parasitol Res. 2014; 113(4):1505-1509.

68. Vitger A. Discovery of Angiostrongylus vasorum (French heartworm) and Crenosoma vulpis (fox lungworm) among dogs in North Copenhagen (Fund af Angiostrongylus vasorum (fransk hjerteorm) og Crenosoma vulpis (raevens lungeorm) blandthundei Nordkobenhavn). Dansk Vet Tidsskr. 2002;85(11):6-13. Danish.

69. Morgan ER, Jefferies R, van Otterdijk L, et al. Angiostrongylus vasorum infection in dogs: Presentation and risk factors. Vet Parasitol. 2010;173(3-4):255-261.

70. Chapman PS, Boag AK, Guitian J, Boswood A. Angiostrongylus vasorum infection in 23 dogs (1999-2002). J Small Anim Pract. 2004;45(9):435-440.

71. Gallagher B, Brennan SF, Zarelli M, Mooney CT. Geographical, clinical, clinicopathological and radiographic features of canine angiostrongylosis in Irish dogs: a retrospective study. Ir Vet J. 2012;65(1):5.

72. Conboy GA. Natural infection of Crenosoma vulpis and Angiostrongylus vasorum in dogs in Atlantic Canada and their treatment with milbemycin oxime. Vet Rec. 2004;155(1):16-18.

73. Bourque A, Conboy G, Miller L, Whitney H, Ralhan S. Angiostrongylus vasorum infection in 2 dogs from Newfoundland. Can Vet J. 2002; 43(11):876-879.

74. Schnyder M, Fahrion A, Ossent P, et al. Larvicidal effect of imidacloprid/ moxidectin spot-on solution in dogs experimentally inoculated with Angiostrongylus vasorum. Vet Parasitol. 2009;166(3-4):326-332.

75. Martin MWS, Ashton G, Simpson VR, Neal C. Angiostrongylosis in Cornwall: clinical presentations of eight cases. J Small Anim Pract. 1993;34(1):20-25.

76. Ridyard A. Heartworm and lungworm in dogs and cat in the UK. In Practice. 2005;27:147-153.

77. Patteson MW, Gibbs C, Wotton PR, Day MJ. Angiostrongylus vasorum infection in seven dogs. Vet Rec. 1993;133(23):565-570.
78. Nicolle AP, Chetboul V, Tessier-Vetzel D, Sampedrano CC, Aletti E, Pouchelon JL. Severe pulmonary arterial hypertension due to Angiostrongylosus vasorum in a dog. Can Vet J. 2006;47(8):792-795.

79. Gredal H, Willesen JL, Jensen HE, et al. Acute neurological signs as the predominant clinical manifestation in four dogs with Angiostrongylus vasorum infections in Denmark. Acta Vet Scand. 2011;53:43.

80. Denk D, Matiasek K, Just FT, et al. Disseminated angiostrongylosis with fatal cerebral haemorrhages in two dogs in Germany: a clinical case study. Vet Parasitol. 2009;160(1-2):100-108.

81. Brennan SF, McCarthy G, McAllister H, Bassett H, Jones BR. Clinical signs, diagnosis and treatment of three dogs with angiostrongylosis in Ireland. Ir Vet J. 2004;57(2):103-109.

82. Wessmann A, Lu D, Lamb CR, et al. Brain and spinal cord haemorrhages associated with Angiostrongylus vasorum infection in four dogs. Vet Rec. 2006;158(25):858-863.

83. Schelling CG, Greene CE, Prestwood AK, Tsang VC. Coagulation abnormalities associated with acute Angiostrongylus vasorum infection in dogs. Am J Vet Res. 1986;47(12):2669-2673.

84. Ramsey IK, Littlewood JD, Dunn JK, Herrtage ME. Role of chronic disseminated intravascular coagulation in a case of canine angiostrongylosis. Vet Rec. 1996;138(15):360-363.

85. Cury MC, Lima WS, Guimarães MP, Carvalho MG. Hematological and coagulation profiles in dogs experimentally infected with Angiostrongylus vasorum (Baillet, 1866). Vet Parasitol. 2002;104(2): 139-149.

86. Gould SM, McInnes EL. Immune-mediated thrombocytopenia associated with Angiostrongylus vasorum infection in a dog. J Small Anim Pract. 1999;40(5):227-232.

87. Whitley NT, Corzo-Menendez N, Carmichael NG, McGarry JW. Cerebral and conjunctival haemorrhages associated with von Willebrand factor deficiency and canine angiostrongylosis. J Small Anim Pract. $2005 ; 46(2): 75-78$

88. Oliveira-Júnior SD, Barçante JM, Barçante TA, Dias SR, Lima WS. Larval output of infected and re-infected dogs with Angiostrongylus vasorum (Baillet, 1866) Kamensky, 1905. Vet Parasitol. 2006;141(1-2): $101-106$.

89. Garosi LS, Platt SR, McConnell JF, Wrayt JD, Smith KC. Intracranial haemorrhage associated with Angiostrongylus vasorum infection in three dogs. J Small Anim Pract. 2005;46(2):93-99.

90. Manning SP. Ocular examination in the diagnosis of angiostrongylosis in dogs. Vet Rec. 2007;160(18):625-627.

91. Mahaffey MB, Losonsky JM, Prestwood AK, Mahaffey EA, Lewis RE. Experimental canine angiostrongylosis: II. Radiographic manifestations. J Am Anim Hosp Assoc. 1981;17:499-502.

92. Boag AK, Lamb CR, Chapman PS, Boswood A. Radiographic findings in 16 dogs infected with Angiostrongylus vasorum. Vet Rec. 2004;154(14):426-430.

93. Dennler M, Makara M, Kranjc A, et al. Thoracic computed tomography findings in dogs experimentally infected with Angiostrongylus vasorum. Vet Radiol Ultrasound. 2011;52(3):289-294.

94. Estèves I, Tessier D, Dandrieux J, et al. Reversible pulmonary hypertension presenting simultaneously with an atrial septal defect and angiostrongylosis in a dog. J Small Anim Pract. 2004;45(4):206-209.

95. Conboy GA. Canine Angiostrongylosis (French heartworm). In: Bowman DD, editor. Companion and Exotic Animal Parasitology. International Veterinary Information Service (http://www.ivis.org); 2000.

96. Barçante JM, Barçante TA, Dias SR, Vieira LQ, Lima WS, Negrão-Corrêa D. A method to obtain axenic Angiostrongylus vasorum first-stage larvae from dog feces. Parasitol Res. 2003;89(2):89-93.

97. Bowman DD. Respiratory system parasites of the dog and cat (part II): trachea and bronchi, and pulmonary vessels. In: Bowman DD, editor. Companion and Exotic Animal Parasitology. International Veterinary Information Service (http://www.ivis.org); 2000.

98. Crawford P, Brooks H, Chantrey J, Gibbons LM. Angiostrongylus vasorum in dogs. Vet Rec 2001;148(8):251-252. 
99. Dodd K. Angiostrongylus vasorum (Baillet, 1866) infestation in a greyhound kennels. Vet Rec. 1973;92(8):195-197.

100. Rosen L, Ash LR, Wallace GD. Life history of the canine lungworm Angiostrongylus vasorum (Baillet). Am J Vet Res. 1970;31(1): 131-143.

101. Ferdushy T, Hasan MT. Angiostrongylus vasorum: the 'French Heartworm'. Parasitol Res. 2010;107(4):765-771.

102. Pinckney RD. Canine Filaroides infection. In: Bowman DD, editor. Companion and Exotic Animal Parasitology. International Veterinary Information Service (http://www.ivis.org); 2000.

103. Prestwood AK, Greene CE, Mahaffey E, Burgess DE. Experimental canine angiostrongylosis: I. Pathologic manifestations. J Am Anim Hosp Assoc. 1981;17:491-497.

104. Barçante JM, Barçante TA, Ribeiro VM, et al. Cytological and parasitological analysis of bronchoalveolar lavage fluid for the diagnosis of Angiostrongylus vasorum infection in dogs. Vet Parasitol. 2008;158(1-2):93-102.

105. Cury MC, Lima SK, Vitor RWA. Enzyme-linked immunosorbent assay (ELISA) for the diagnosis of Angiostrongylus vasorum (Baillet, 1866) infection in dogs. Rev Méd Vét. 1996;147(7):525-530.

106. Cury MC, Guimarães MP, Lima WS, Vitor RW. Western blot analysis of the humoral response of dogs experimentally infected with Angiostrongylus vasorum (Baillet, 1866). Vet Parasitol. 2002;106(1):83-87.

107. De Oliveira Vasconcelos V, De Almeida Vitor RW, Dos Santos Lima W. Identification of stage-specific proteins of Angiostrongylus vasorum (Baillet, 1866) Kamensky. Parasitol Res. 2008;102(3):389-395.

108. Verzberger-Epshtein I, Markham RJ, Sheppard JA, Stryhn H, Whitney H, Conboy GA. Serologic detection of Angiostrongylus vasorum infection in dogs. Vet Parasitol. 2008;151(1):53-60.

109. Schnyder M, Tanner I, Webster P, Barutzki D, Deplazes P. An ELISA for sensitive and specific detection of circulating antigen of Angiostrongylus vasorum in serum samples of naturally and experimentally infected dogs. Vet Parasitol. 2011;179(1-3):152-158.

110. Schucan A, Schnyder M, Tanner I, Barutzki D, Traversa D, Deplazes P. Detection of specific antibodies in dogs infected with Angiostrongylus vasorum. Vet Parasitol. 2012;185(2-4):216-224.
111. Caldeira RL, Carvalho OS, Mendonça CL, et al. Molecular differentiation of Angiostrongylus costaricensis, A. cantonensis, and A. vasorum by polymerase chain reaction-restriction fragment length polymorphism. Mem Inst Oswaldo Cruz. 2003;98(8):1039-1043.

112. Jefferies R, Morgan ER, Shaw SE. A SYBR green real-time PCR assay for the detection of the nematode Angiostrongylus vasorum in definitive and intermediate hosts. Vet Parasitol. 2009;166(1-2): 112-118.

113. Willesen JL, Kristensen AT, Jensen AL, Heine J, Koch J. Efficacy and safety of imidacloprid/moxidectin spot-on solution and fenbendazole in the treatment of dogs naturally infected with Angiostrongylus vasorum (Baillet, 1866). Vet Parasitol. 2007;147(3-4):258-264.

114. Conboy G, Schenker R, Strehlau G. Efficacy of Milbemax (milbemycin/ praziquantel) for the treatment and prevention of Angiostrongylus vasorum infection in dogs. In: Proceedings of the Joint 49th Annual Meeting of the American Association of Veterinary Parasitologists/79th Meeting of the American Society of Parasitologists; July 24-28, 2004; Philadelphia, PA, USA. p 92. Abs 121.

115. Søland J, Bolt G. Hypovolaemic shock after anthelmintic treatment of canine angiostrongylosis. J Small Anim Pract. 1996;37(12): 594-596.

116. Böhm C, Schnyder M, Thamsborg SM, et al. Assessment of the combination of spinosad and milbemycin oxime in preventing the development of canine Angiostrongylus vasorum infections. Vet Parasitol. 2014;199(3-4):272-277.

117. Braga FR, Carvalho RO, Araujo JM, et al. Predatory activity of the fungi Duddingtonia flagrans, Monacrosporium thaumasium, Monacrosporium sinense and Arthrobotrys robusta on Angiostrongylus vasorum first-stage larvae. J Helminthol. 2009;83(4):303-308.

118. Gasser RB, Jabbar A, Mohandas N, et al. Mitochondrial genome of Angiostrongylus vasorum: comparison with congeners and implications for studying the population genetics and epidemiology of this parasite. Infect Genet Evol. 2012;12(8):1884-1891.

119. Ansell BR, Schnyder M, Deplazes P, et al. Insights into the immuno-molecular biology of Angiostrongylus vasorum through transcriptomics - prospects for new interventions. Biotechnol Adv. 2013;31(8):1486-1500.
Veterinary Medicine: Research and Reports

\section{Publish your work in this journal}

Veterinary Medicine: Research and Reports is an international, peer-reviewed, open access journal publishing original research, case reports, editorials, reviews and commentaries on all areas of veterinary medicine. The manuscript management system is completely online and includes a very quick and fair peer-review system.

\section{Dovepress}

Visit http://www.dovepress.com/testimonials.php to read real quotes from published authors. 